\title{
molecules
}

ISSN 1420-3049

Article

www.mdpi.com/journal/molecules

\section{Direct One-Pot Synthesis of Primary 4-Amino-2,3-diaryl- quinolines via Suzuki-Miyaura Cross-Coupling of 2-Aryl-4-azido-3-iodoquinolines with Arylboronic Acids}

\author{
Malose Jack Mphahlele * and Mamasegare Mabel Mphahlele \\ Department of Chemistry, College of Science, Engineering and Technology, University of South Africa, \\ P.O. Box 392, Pretoria 0003, South Africa
}

* Author to whom correspondence should be addressed; E-Mail: mphahmj@unisa.ac.za;

Tel. +27-12-429-8805; Fax: +27-12-429-8549.

Received: 30 September 2011; in revised form: 20 October 2011 / Accepted: 21 October 2011 /

Published: 25 October 2011

\begin{abstract}
Palladium-catalyzed Suzuki-Miyaura cross-coupling of 2-aryl-4-azido-3-iodoquinolines with arylboronic acids afforded the corresponding primary 4-amino-2,3diarylquinolines in a single-pot operation along with symmetrical biaryls and traces of the 2,3-diaryl-4-azidoquinolines. A plausible mechanism, which implicates palladium hydride species in the reduction of the incipient 2,3-diaryl-4-azidoquinolines to afford the 4-amino2,3-diarylquinolines is proposed.
\end{abstract}

Keywords: 2-aryl-4-azido-3-iodoquinolines; Suzuki-Miyaura cross-coupling; symmetrical biaryls; 4-azido-2,3-diarylquinolines; 4-amino-2,3-diarylquinolines

\section{Introduction}

Our continued interest in the synthesis of primary 4-aminoquinoline derivatives stems from their importance as antimalarial, anti-inflammatory, antibacterial, and antihypertensive agents [1-4] as well as immunostimulants and non-nucleoside HIV-1 inhibitors [5,6]. Aryl substituted quinoline derivatives are also known to serve as potent inhibitors of tyrosine kinase PDGF-RTK [7]. Moreover, the 4-amino2-arylquinolines have also been found to represent a novel class of NR1/2B subtype selective $N$-methyl-D-aspartate (NMDA) receptor antagonists [8]. Although there are several methods described in the literature for the synthesis of primary 4-amino-2-arylquinolines [6,9-13], corresponding data for the 
synthesis of 2,3-disubstituted 4-aminoquinoline derivatives is considerably less well documented $[13,14]$. These polysubstituted quinoline derivatives are not accessible via classical methods such as the Skraup, Doebner-von Miller, Friedlander and Combes syntheses [15]. Consequently, an indirect approach to efficiently functionalize the presynthesized halogenated quinoline derivatives via nucleophilic displacement and/or metal-catalyzed cross-coupling reactions leading to $\mathrm{Cs} p^{2}-\mathrm{N}$ and/or $\mathrm{C} s p^{2}-\mathrm{Cs} p^{2}$ bond formation remains the method of choice. The 4-(2-methylphenylamino)-3-iodoquinolines, for example, were previously subjected to palladium-catalyzed Heck reaction with terminal alkenes to afford the corresponding 3-vinylquinolines with gastric $\mathrm{H}^{+} / \mathrm{K}^{+}$-ATPase inhibitory activity [16]. On the other hand, the analogous 2-aryl-3-iodo-4-(phenylamino)quinolines were found to undergo one-pot palladium mediated $\mathrm{C}-\mathrm{I}$ and $\mathrm{C}-\mathrm{H}$ bond activation and subsequent Suzuki-Miyaura cross-coupling with arylboronic acids to afford mixtures of the 2,3-diaryl-4-(phenylamino)quinolines and 2-aryl-4-([(1,1'-biaryl)-2-yl]amino)quinoline derivatives [17]. Hitherto this investigation, the Staudinger reaction of 2-aryl-4-azido-3-halogenoquinolines $(\mathrm{X}=\mathrm{Br}$, I) with triphenylphosphine in refluxing tetrahydrofuran afforded the corresponding 2-aryl-3-halogeno-4-(triphenylphosphoranylideneamino)quinolines [18]. The latter were either hydrolyzed to the corresponding 4-amino-2-aryl3-halogenoquinolines or subjected to palladium-catalyzed Suzuki-Miyaura cross-coupling with phenylboronic acid followed by acetic acid-promoted hydrolysis of the incipient 2,3-diarylphosphazenes to afford the 4-amino-2,3-diarylquinolines [18].

A literature search revealed two papers describing the outcome of tetrakis(triphenylphosphine)palladium $(0)\left[\mathrm{Pd}\left(\mathrm{PPh}_{3}\right)_{4}\right]$-catalyzed Suzuki-Miyaura cross-couplings of 4-azido-3-bromopyridine with heteroarylboronic acids [19] and 1-azido-2-bromobenzene with a series of aryl- and heteroarylboronic acids [20]. In one case, involving the coupling of 1-azido-2-bromobenzene with 2-thiopheneboronic acid, the authors isolated 2-(2-azidophenyl)thiophene (40\%), bromoaniline (2\%) and 2-thiophenylaniline $(3 \%)$ as products [20]. They attributed the formation of bromoaniline and 2-thiophenylaniline to hydrolysis of the corresponding incipient iminophosphorane resulting from the reaction between triphenylphosphine ligand and the azide function [20]. Prompted by this literature observation and the ability of iodine to facilitate metal-catalyzed carbon-carbon bond formation of the 2-aryl-3-iodo-4(triphenylphosphoranylideneamino)quinolines [18] we decided to investigate the reactivity of the known 2-aryl-4-azido-3-iodoquinolines in palladium-catalyzed Suzuki-Miyaura cross-couplings. The main aim of this investigation was to assess the possibility of effecting direct one-pot synthesis of the primary 4-amino-2,3-diarylquinolines via Suzuki-Miyaura cross-coupling of the 2-aryl-4-azido-3iodoquinolines with arylboronic acids.

\section{Results and Discussion}

The Suzuki-Miyaura reaction of aryl or heteroaryl halides with arylboronic acids is a well established procedure and its mechanism generally involves: (i) oxidative addition of an aryl halide to a $\mathrm{Pd}(0)$ active catalyst; (ii) transmetallation of $\mathrm{Ar}-\mathrm{Pd}-\mathrm{X}$ with $\operatorname{Ar}{ }^{\prime} \mathrm{B}(\mathrm{OH})_{3}{ }^{-} \mathrm{M}^{+}$; and (iii) reductive elimination to give a biaryl product [21,22]. The efficiency of a palladium catalyst, on the other hand, depends strongly on the ligand of palladium atom and the overall reactivity also depends on the nature of the palladium $(0)$ complex precursor $[23,24]$. With this consideration in mind, we reacted the 4-azido-2-aryl-3-iodoquinolines 1a,b with phenylboronic acid (1.2 equiv.) in refluxing DMF in the 
presence of $\mathrm{Pd}\left(\mathrm{PPh}_{3}\right)_{4}$ and $2 \mathrm{M} \mathrm{K}_{2} \mathrm{CO}_{3}$ as a base as a reference starting point for exploration based on literature precedents. After $48 \mathrm{~h}$ we isolated by column chromatography on silica gel three products in sequence, which were identified using a combination of spectroscopic techniques as the symmetrical biphenyl 2a (3\%, 5\%) 2,3-diaryl-4-azidoquinolines 3a (6\%), b (7\%) and 4-amino-2,3-diarylquinolines 4a $(15 \%)$, b (35\%), respectively. Under similar reaction conditions using palladium acetate as pre-catalyst, phenylboronic acid (1.5-2 equiv.) and $2 \mathrm{M} \mathrm{K}_{2} \mathrm{CO}_{3}$ as a base and DMF as solvent, we also isolated by column chromatography on silica gel after $48 \mathrm{~h}$ the biphenyl 2a (21\%, 26\%), 4-azido-2,3diarylquinolines 3c (14\%), d (14\%) and 4-amino-2,3-diarylquinolines $4 \mathbf{c}(30 \%)$, d (50\%) in sequence. Analytical data for products 4 was found to compare favourably with those of the corresponding derivatives prepared as described in our previous communication [18]. The ${ }^{1} \mathrm{H}-\mathrm{NMR}$ and ${ }^{13} \mathrm{C}-\mathrm{NMR}$ spectra of the azido derivatives $\mathbf{3}$, on the other hand, revealed the presence of an increased number of signals in the aromatic region, which distinguished these products from the corresponding precursors. The presence of strong IR absorption band in the $v_{\max } 2,110-2,119 \mathrm{~cm}^{-1}$ (asymmetric) and $v_{\max }$ 1,220-1,260 $\mathrm{cm}^{-1}$ (symmetric) regions further distinguished systems $\mathbf{3}$ from the amino derivatives 4 . The analogous 4-phenyl-5-azidoquinolines on the other hand, previously afforded in refluxing xylene pyrido[2,3,4- $k l$ ] acridines via an intramolecular nitrene insertion reaction [25]. The 3-aryl-4-azido-7methoxyquinolin-2(1H)-ones prepared from the reaction of 3-aryl-4-(chloro/tosyloxy)-7-methoxyquinolin-2(1H)-ones with sodium azide in refluxing DMF were also found to undergo thermolytic ring closure to afford the 5-alkyl-3-methoxy-11H-indolo[3,2-c]quinolin-6(5H)-ones [26]. In the current investigation, no products resulting from thermolytic ring closure of $\mathbf{3}$ were isolated from the reaction mixtures.

Despite the outcome of the above reaction, we were concerned about the low yields and prolonged reaction times, presumably due to the slow oxidative addition step using $\mathrm{Pd}\left(\mathrm{PPh}_{3}\right)_{4}$ as precursor of the palladium(0) complex. This slow oxidative addition step is attributed to the inhibiting role of the extra $\mathrm{PPh}_{3}$ generated in the second equilibrium $\left\{\mathrm{SPd}(0)\left(\mathrm{PPh}_{3}\right)_{3} \rightleftharpoons S \mathrm{Pd}(0)\left(\mathrm{PPh}_{3}\right)_{2}+\mathrm{PPh}_{3}\left(K_{2} /\left[\mathrm{PPh}_{3}\right]<<1\right)\right.$; $S=$ solvent $\}$ to afford the low reactivity ligated 14-electron species $\left(\operatorname{Pd}(0)\left(\mathrm{PPh}_{3}\right)_{2}\right)$ [24]. Conversely, the oxidative addition performed by the palladium( 0$)$ complex $\left(\mathrm{Pd}(0)\left(\mathrm{PPh}_{3}\right)_{2} \mathrm{Cl}^{-}\right)$generated by the reduction of dichlorobis(triphenylphosphine)palladium(II) $\left(\mathrm{PdCl}_{2}\left(\mathrm{PPh}_{3}\right)_{2}\right)$ is reported to be more than 30 times faster than that performed from $\mathrm{Pd}(0)\left(\mathrm{PPh}_{3}\right)_{4}$ [24]. Likewise, alkylphosphine ligands are known to coordinate with palladium and increase its electron density more than arylphosphines and, in turn, accelerate the oxidative addition and reductive elimination steps in the catalytic cycle [27,28]. Consequently, we subjected substrates 1a-d to 2 equiv. of phenylboronic acid in the presence of $\mathrm{PdCl}_{2}\left(\mathrm{PPh}_{3}\right)_{2}$-tricyclohexylphosphine $\left(\mathrm{PCy}_{3}\right)$ catalyst mixture and $2 \mathrm{M}$ potassium carbonate in DMF under reflux (Scheme 1). The reaction in the presence of $\mathrm{PdCl}_{2}\left(\mathrm{PPh}_{3}\right)_{2}-\mathrm{PCy}_{3}$ catalyst mixture was complete within $18 \mathrm{~h}$. Analysis of the crude product mixtures by thin layer chromatography revealed in all cases three spots of different polarity and intensity with no traces of the spot corresponding to the starting material. The mixture was isolated by column chromatography on silica gel to afford the biphenyl 2a, 4-azido-2,3-diarylquinolines 3a-d (minor) and 4-amino-2,3-diarylquinolines 4a-d (major) in sequence. The reaction conditions were also extended to include 4-fluorophenylboronic and 4-methoxyphenylboronic acids as coupling partners. Although in all cases, traces of the 4-azido-2,3diarylquinolines 3 (2nd spot) were detected by thin layer chromatography in the crude product mixture, 
careful column chromatographic separation on silica gel in most cases led to isolation of the self-coupled biaryl derivatives $\mathbf{2 b , c}$ (minor) and the 4-amino-2,3-diarylquinolines $\mathbf{4 a - 1}$ as the major products.

Scheme 1. $\mathrm{PdCl}_{2}\left(\mathrm{PPh}_{3}\right)_{2}-\mathrm{PCy}$ catalyzed Suzuki-Miyaura cross-coupling of 1 with $\mathrm{ArB}(\mathrm{OH})_{2}$.

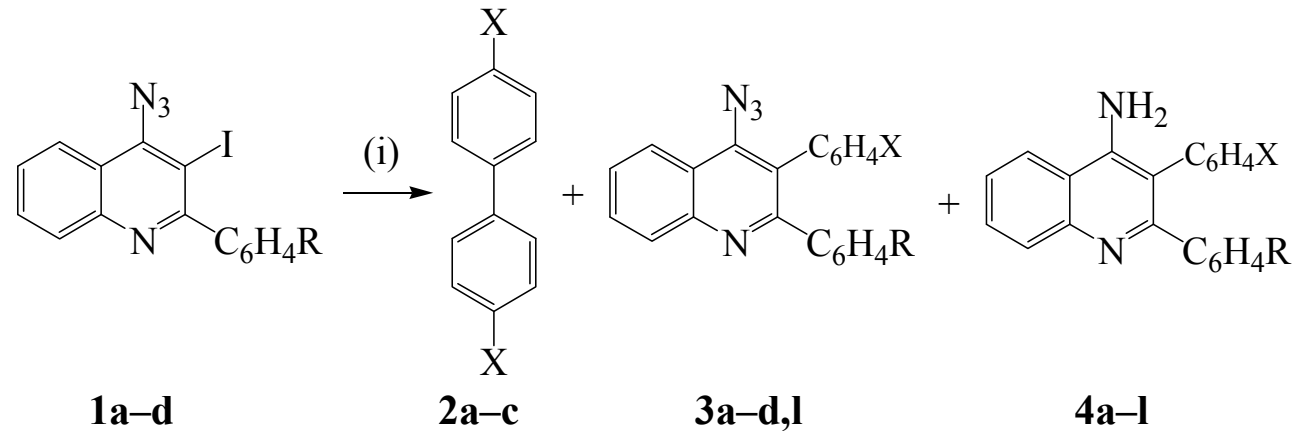

Reagents and conditions: (i) $\mathrm{ArB}(\mathrm{OH})_{2}$ (2.0 equiv.), $\mathrm{PdCl}_{2}\left(\mathrm{PPh}_{3}\right)_{2}, \mathrm{PCy}_{3}, 2 \mathrm{M} \mathrm{K}_{2} \mathrm{CO}_{3}$, DMF, heat, $18 \mathrm{~h}$.

\begin{tabular}{cccccc}
\hline $\mathbf{3 / 4}$ & 4-R & 4-X & \% Yield 2 & \% Yield 3 & \% Yield 4 \\
\hline $\mathbf{a}$ & $4-\mathrm{H}$ & $4-\mathrm{H}$ & $24(\mathbf{2 a})$ & 12 & 57 \\
$\mathbf{b}$ & $4-\mathrm{F}$ & $4-\mathrm{H}$ & $10(\mathbf{2 a})$ & 16 & 65 \\
$\mathbf{c}$ & $4-\mathrm{Cl}$ & $4-\mathrm{H}$ & $15(\mathbf{2 a})$ & 10 & 54 \\
$\mathbf{d}$ & $4-\mathrm{OMe}$ & $4-\mathrm{H}$ & $26(\mathbf{2 a})$ & 11 & 66 \\
$\mathbf{e}$ & $4-\mathrm{H}$ & $4-\mathrm{F}$ & $20(\mathbf{2 b})$ & - & 65 \\
$\mathbf{f}$ & $4-\mathrm{F}$ & $4-\mathrm{F}$ & $12(\mathbf{2 b})$ & - & 66 \\
$\mathbf{g}$ & $4-\mathrm{Cl}$ & $4-\mathrm{F}$ & $14(\mathbf{2 b})$ & - & 56 \\
$\mathbf{h}$ & $4-\mathrm{OMe}$ & $4-\mathrm{F}$ & $21(\mathbf{2 b})$ & - & 64 \\
$\mathbf{i}$ & $4-\mathrm{H}$ & $4-\mathrm{MeO}$ & $17(\mathbf{2 c})$ & - & 63 \\
$\mathbf{j}$ & $4-\mathrm{F}$ & $4-\mathrm{MeO}$ & $18(\mathbf{2 c})$ & - & 57 \\
$\mathbf{k}$ & $4-\mathrm{Cl}$ & $4-\mathrm{MeO}$ & $13(\mathbf{2 c})$ & - & 60 \\
$\mathbf{l}$ & $4-\mathrm{OMe}$ & $4-\mathrm{MeO}$ & $28(\mathbf{2 c})$ & 9 & 68 \\
\hline
\end{tabular}

At first glance, we also thought products 4 are the result of the initial cross-coupling of 1 with arylboronic acids and subsequent in situ Staudinger reaction of the 2,3-diaryl-4-azidoquinolines 3 with $\mathrm{PPh}_{3}$ released from the catalyst followed by hydrolysis of the incipient 2,3-diaryl-4-(triphenylphosphoranylideneamino)quinolones, in analogy with the previous literature observation [20]. However, this possibility was ruled out by the absence of triphenylphosphonium oxide in the reaction mixture or crude product (tlc monitoring or ${ }^{31} \mathrm{P}-\mathrm{NMR}$ ), which is the expected by-product of hydrolysis of phosphazene derivatives [18]. Recourse to literature, revealed a paper describing the results of palladium acetate $\left(\mathrm{Pd}(\mathrm{OAc})_{2}\right)$-catalyzed Suzuki-Miyaura cross-coupling of nitroaryl halides with arylboronic acids in $\mathrm{DMF} / \mathrm{H}_{2} \mathrm{O}$ at $150{ }^{\circ} \mathrm{C}$ using $\mathrm{K}_{2} \mathrm{CO}_{3}$ as a base in the absence or presence of a ligand $\left(\mathrm{PPh}_{3}\right.$ or $\left.\mathrm{DABCO}\right)$ [29]. The reaction afforded the corresponding biaryl derivatives with simultaneous reduction of nitro- to amino group and the authors attributed the reduction of the nitro group to molecular hydrogen based on literature precedent [30]. However, DMF-water mixture failed to reduce nitrobenzene to aniline at $150{ }^{\circ} \mathrm{C}$ [29]. Moreover, $\mathrm{PdCl}_{2}\left(\mathrm{PPh}_{3}\right)_{2}-\mathrm{PCy}_{3}$ catalyzed cross-coupling of 1d with $\mathrm{PhB}(\mathrm{OH})_{2}$ using $2 \mathrm{M} \mathrm{K}_{2} \mathrm{CO}_{3}$ in dioxane also afforded $\mathbf{2 a}(26 \%)$, 3d (11\%) and 4d (62\%) in sequence. We envisioned that molecular hydrogen generated from DMF-water medium in the presence of $\mathrm{Pd}\left(\mathrm{PPh}_{3}\right)_{4}$ or $\mathrm{PdCl}_{2}\left(\mathrm{PPh}_{3}\right)_{2}$ would also hydrogenolyze the azidoiodoquinolines $\mathbf{1}$ in analogy with 
literature observation for the selective hydrogenolysis of azidoiodoarenes by $\mathrm{H}_{2}-\mathrm{Pd} / \mathrm{C}$ mixture to afford the azidoarenes [31].

The intriguing results observed in this investigation prompted us to propose a mechanism outlined in Scheme 2 to account for the one-pot palladium-catalyzed cross-coupling and subsequent reduction of the azido group to afford the primary 4-aminoquinolines 4 . The symmetrical biaryls 2 are the result of the self-coupling of aryl groups from arylboronic acid. Homo-coupling of arylboronic acids is a side reaction usually observed for the Suzuki-Miyaura cross-coupling reactions under both $\mathrm{Pd}\left(\mathrm{PPh}_{3}\right)_{4}$ and $\mathrm{Pd}(\mathrm{OAc})_{2}$ catalysis especially when the cross-coupling is very slow [32,33]. The self-coupling step is known to be accompanied by the release of palladium hydride $\left(\mathrm{PdH}_{2}\right)$ along with metaboric acid $(\mathrm{HOB}=\mathrm{O})$ liberated in the form of borate under alkaline aqueous medium used in the Suzuki-Miyaura cross-coupling reactions [32]. The intermediate palladium hydride released during the catalytic cycle may either release hydrogen or serve as hydride source to reduce oxidants present in the reaction media and generate $\operatorname{Pd}(0)$ [32]. Although palladium hydride is implicated in the self-coupling mechanism [32], palladium hydrides $\mathrm{L}_{2} \mathrm{PdHCl}\left[\mathrm{L}=\mathrm{PCy}_{3}\right.$ or $\left.\mathrm{P}(t-\mathrm{Bu})_{3}\right]$, have been observed during the course of palladium-catalyzed Heck reaction [34].

Scheme 2. Proposed mechanism for the one-pot Suzuki-Miyaura cross-coupling and reduction of 1 incorporating self-coupling of $\mathrm{ArB}(\mathrm{OH})_{2}$.

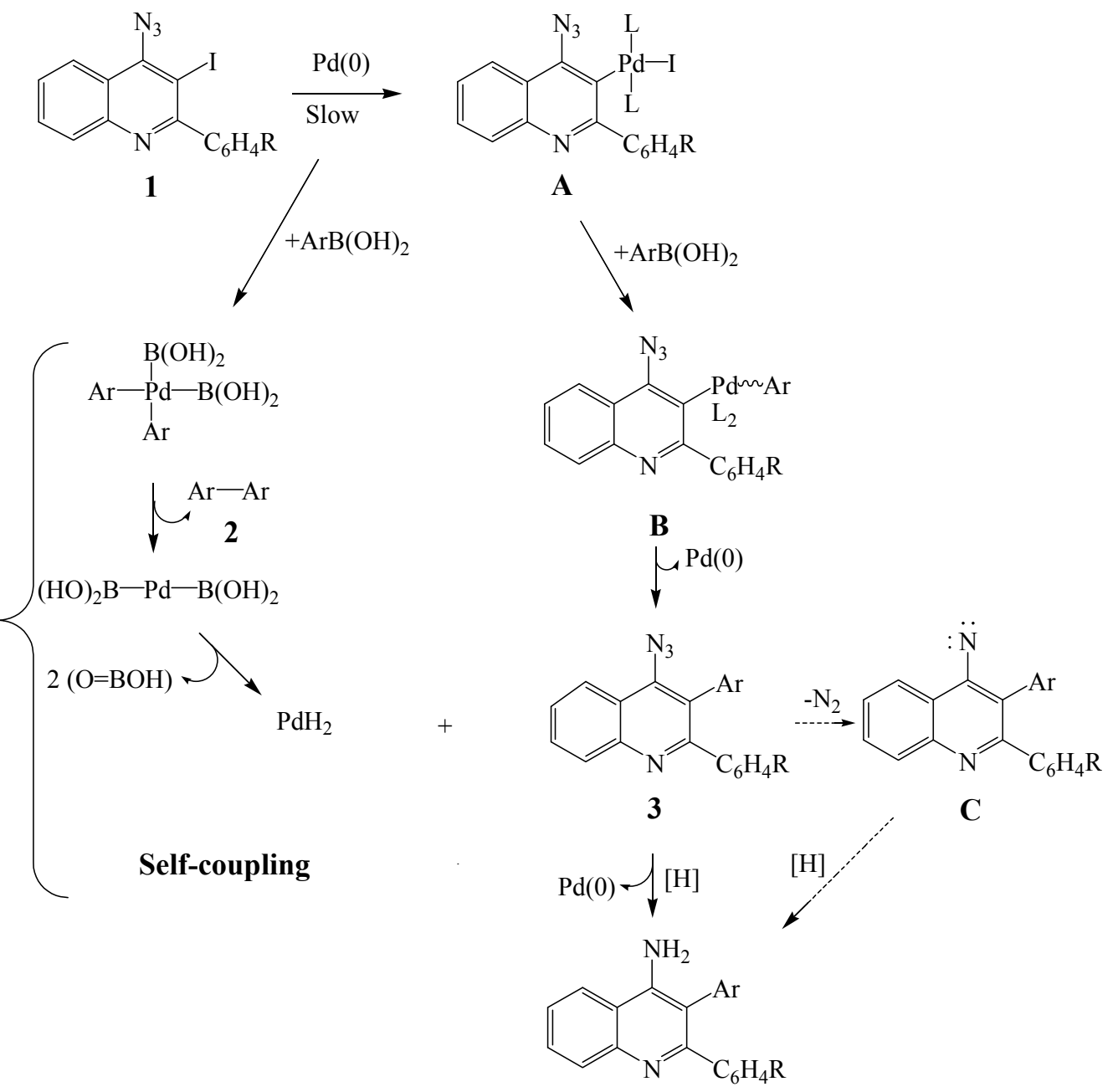


The envisioned self-coupling step is presumably accompanied by a slow oxidative addition of palladium(0) complex into $\mathbf{1}$ to form $\mathbf{A}$, followed by transmetallation and reductive elimination from $\mathbf{B}$ to afford the corresponding 2,3-diaryl-4-azidoquinoline $\mathbf{3}$ (detected by tlc or isolated by column chromatography) as invoked in the classical Suzuki-Miyaura cross-coupling reaction mechanism. We envision that intermediate 3 is reduced by palladium hydride $\left(\mathrm{PdH}_{2}\right.$ or $\left.\mathrm{L}_{2} \mathrm{PdHI}\right)$ released from the self-coupling reaction to afford the 4-amino-2,3-diarylquinoline 4 in moderate yields. The possibility of formation of the latter via reduction of a nitrene intermediate $\mathbf{C}$ generated from $\mathbf{3}$ cannot be completely ruled out. Despite the fact that our proposed mechanism is necessarily speculative, it represents the best option consistent with the formation of the observed products in the presence or absence of $\mathrm{PCy}_{3}$.

\section{Experimental}

\subsection{General}

Melting points were recorded on a Thermocouple digital melting point apparatus and are uncorrected. IR spectra were recorded as powders using a FTS 7000 Series Digilab Win-IR Pro ATR (attenuated total reflectance) spectrometer. For column chromatography, Merck kieselgel 60 $(0.063-0.200 \mathrm{~mm})$ was used as stationary phase. NMR spectra were obtained as $\mathrm{CDCl}_{3}$ solutions using Varian Mercury $300 \mathrm{MHz}$ NMR spectrometer and the chemical shifts are quoted relative to the solvent peaks. Low- and high-resolution mass spectra were recorded at an ionization potential of $70 \mathrm{eV}$ using Micromass Autospec-TOF (double focusing high resolution) instrument. The synthesis and characterization of substrates 1 have been described elsewhere [18].

\subsection{Typical Procedure for the $\mathrm{PdCl}_{2}\left(\mathrm{PPh}_{3}\right)_{2}-\mathrm{PC} y_{3}$ Catalyzed Cross-Coupling Reactions of 1 with $\mathrm{ArB}(\mathrm{OH})_{2}$}

\subsubsection{Biphenyl (2a), 4-Azido-2,3-diphenylquinoline (3a) and 4-Amino-2,3-diphenylquinoline (4a)}

A mixture of $1 \mathrm{a}(0.20 \mathrm{~g}, 0.54 \mathrm{mmol})$, phenylboronic acid $(0.13 \mathrm{~g}, 1.08 \mathrm{mmol}), 2 \mathrm{M} \mathrm{K}_{2} \mathrm{CO}_{3}(1.2 \mathrm{~mL})$, $\mathrm{PdCl}_{2}\left(\mathrm{PPh}_{3}\right)_{2}(0.02 \mathrm{~g}, 0.03 \mathrm{mmol})$ and $\mathrm{PCy}_{3}(0.02 \mathrm{~g}, 0.05 \mathrm{mmol})$ in DMF $(5 \mathrm{~mL})$ in a two-necked flask equipped with a stirrer bar, rubber septum and a condenser was flushed for 30 min with argon gas. A balloon filled with argon gas was connected to the top of the condenser and the mixture was heated with stirring at $80-90{ }^{\circ} \mathrm{C}$ under argon atmosphere for $18 \mathrm{~h}$ and then allowed to cool to room temperature. The cooled mixture was poured into a ice-cold water and the product was taken-up into chloroform. The combined organic extracts were washed with brine, dried over anhydrous $\mathrm{MgSO}_{4}$, filtered and then evaporated under reduced pressure. The residue was purified by column chromatography on silica gel (20\% ethyl acetate-hexane) to afford $\mathbf{2 a}, \mathbf{3 a}$ and $\mathbf{4 a}$ in sequence.

Biphenyl (2a). Solid (19.5 mg, 24\%), mp 69-71 ${ }^{\circ} \mathrm{C}$ (ethanol) (Lit. [35] 70-72 ${ }^{\circ} \mathrm{C}$ ), $R_{\mathrm{F}} 0.94$.

4-Azido-2,3-diphenylquinoline (3a). Solid (20 mg, 12\%) $\mathrm{mp} 125-127^{\circ} \mathrm{C}, R_{\mathrm{F}} 0.63 ; v_{\max }$ (neat) 764, 838, $1174,1240,1379,2110 \mathrm{~cm}^{-1} ; \delta_{\mathrm{H}}\left(300 \mathrm{MHz}, \mathrm{CDCl}_{3}\right) 7.19-7.22(\mathrm{~m}, 3 \mathrm{H}), 7.25-7.35(\mathrm{~m}, 6 \mathrm{H}), 7.55-7.60$ $(\mathrm{m}, 2 \mathrm{H}), 7.76(\mathrm{dt}, J 1.5$ and $7.8 \mathrm{~Hz}, 1 \mathrm{H}), 8.15(\mathrm{dd}, J 0.6$ and $8.7 \mathrm{~Hz}, 1 \mathrm{H}), 8.23(\mathrm{~d}, J 8.7 \mathrm{~Hz}, 1 \mathrm{H}) ; \delta_{\mathrm{C}}(75$ $\mathrm{MHz}, \mathrm{CDCl}_{3}$ ) 123.0, 126.5, 126.8, 127.7, 127.8, 128.3, 128.4, 129.0, 129.4, 129.6, 130.3, 131.5, 134.5, 
140.1, 142.4, 147.8, 159.6; m/z $295\left(100, \mathrm{MH}^{+}-\mathrm{N}_{2}\right)$, HRMS (ES): $\mathrm{MH}^{+}-\mathrm{N}_{2}$ found 295.1237. $\mathrm{C}_{21} \mathrm{H}_{15} \mathrm{~N}_{2}^{+}$ requires 295.1235 .

4-Amino-2,3-diphenylquinoline (4a). Solid (90 mg, 57\%), mp 238-240 ${ }^{\circ} \mathrm{C}\left(\right.$ Lit. [18] 239-241 $\left.{ }^{\circ} \mathrm{C}\right), R_{\mathrm{F}} 0.15$.

3.2.2. Biphenyl (2a), 4-Azido-2-(4-fluorophenyl)-3-phenylquinoline (3b) and 4-Amino-2-(4-fluoro-phenyl)-3-phenylquinoline (4b)

A mixture of $\mathbf{1 b}(0.50 \mathrm{~g}, 1.28 \mathrm{mmol})$, phenylboronic acid $(0.31 \mathrm{~g}, 2.56 \mathrm{mmol}), \mathrm{PdCl}_{2}\left(\mathrm{PPh}_{3}\right)_{2}(0.04 \mathrm{~g}$, $0.06 \mathrm{mmol}), \mathrm{PCy}_{3}(0.04 \mathrm{~g}, 0.13 \mathrm{mmol}), 2 \mathrm{M} \mathrm{K}_{2} \mathrm{CO}_{3}(2.6 \mathrm{~mL})$ in DMF $(10 \mathrm{~mL})$ was treated as described above. Work-up and column chromatography on silica gel (20\% ethyl acetate-hexane) afforded $2 \mathbf{a}(19.6 \mathrm{mg}, 10 \%), R_{\mathrm{F}} 0.90 ; \mathbf{3 b}$ and $\mathbf{4 b}$ in sequence.

4-Azido-2-(4-fluorophenyl)-3-phenylquinoline (3b). Solid (69.6 mg, $16 \%) \mathrm{mp} 131-133{ }^{\circ} \mathrm{C}, R_{\mathrm{F}} 0.63$; $v_{\max }$ (neat) 764, 838, 1159, 1221, 1364, 1480, $2110 \mathrm{~cm}^{-1} ;{ }^{1} \mathrm{H}-\mathrm{NMR} \delta_{\mathrm{H}}\left(300 \mathrm{MHz}, \mathrm{CDCl}_{3}\right) 6.90(\mathrm{t}, J 8.7 \mathrm{~Hz}$, 2H), 7.24-7.30 (m, 4H), 7.35-7.38 (m, 3H), 7.59 (dt, $J 1.2$ and 7.5 Hz, 1H), 7.77 (dt, $J 1.5$ and 7.8 Hz, $1 \mathrm{H}), 8.13(\mathrm{~d}, J 8.4 \mathrm{~Hz}, 1 \mathrm{H}), 8.23(\mathrm{dd}, J 1.2$ and $8.4 \mathrm{~Hz}, 1 \mathrm{H}) ;{ }^{13} \mathrm{C}-\mathrm{NMR} \delta_{\mathrm{C}}\left(75 \mathrm{MHz}, \mathrm{CDCl}_{3}\right) 114.7(\mathrm{~d}$, $\left.{ }^{2} J_{\mathrm{CF}} 21.6 \mathrm{~Hz}\right), 121.5,123.0,126.3,126.9,128.5,128.6,129.4,130.4,131.4,131.5\left(\mathrm{~d},{ }^{3} J_{\mathrm{CF}} 8.3 \mathrm{~Hz}\right)$, 134.4, $136.2\left(\mathrm{~d},{ }^{4} J_{\mathrm{CF}} 3.6 \mathrm{~Hz}\right), 142.6,147.8,158.5,162.4$ (d, $\left.{ }^{1} J_{\mathrm{CF}} 246.2 \mathrm{~Hz}\right) ; m / z 313\left(100, \mathrm{MH}^{+}-\mathrm{N}_{2}\right)$, HRMS (ES): $\mathrm{MH}^{+}-\mathrm{N}_{2}$ found 313.1146. $\mathrm{C}_{21} \mathrm{H}_{14} \mathrm{FN}_{2}{ }^{+}$requires 313.1141.

4-Amino-2-(4-fluorophenyl)-3-phenylquinoline (4b). Solid (260 mg, 65\%), mp 225-228 ${ }^{\circ} \mathrm{C}$ (Lit. [18] $\left.215-217^{\circ} \mathrm{C}\right), R_{\mathrm{F}} 0.23$.

3.2.3. Biphenyl (2a), 4-Azido-2-(4-chlorophenyl)-3-phenylquinoline (3c) and 4-Amino-2-(4-chloro-phenyl)-3-phenylquinoline (4c)

A mixture of $1 \mathrm{c}(0.25 \mathrm{~g}, 0.62 \mathrm{mmol})$, phenylboronic acid $(0.15 \mathrm{~g}, 1.23 \mathrm{mmol}), \mathrm{PdCl}_{2}\left(\mathrm{PPh}_{3}\right)_{2}(0.02 \mathrm{~g}$, $0.03 \mathrm{mmol}), \mathrm{PCy}_{3}(0.20 \mathrm{~g}, 0.06 \mathrm{mmol}), 2 \mathrm{M} \mathrm{K}_{2} \mathrm{CO}_{3}(1.2 \mathrm{~mL})$ in DMF (6 mL) was treated as described above. Work-up and column chromatography on silica gel (20\% ethyl acetate-hexane) afforded 2a $(20.2 \mathrm{mg}, 21 \%), R_{\mathrm{F}} 0.90, \mathbf{3 c}$ and $\mathbf{4 c}$ in sequence.

4-Azido-2-(4-chlorophenyl)-3-phenylquinoline (3c). Solid (19 mg, 10\%), mp 140-141 ${ }^{\circ} \mathrm{C}$ (ethanol), $R_{\mathrm{F}}$ (20\% ethyl acetate-hexane) $0.54 ; v_{\max }$ (neat) 838, 1159, 1221, 1364, 1504, $2110 \mathrm{~cm}^{-1} ;{ }^{1} \mathrm{H}-\mathrm{NMR} \delta_{\mathrm{H}}$ $\left(300 \mathrm{MHz}, \mathrm{CDCl}_{3}\right)$ 7.16-7.20 (m, 2H), 7.22-7.28 (m, 4H), 7.33-7.38 (m, 3H), 7.58 (dt, $J 1.2$ and $8.1 \mathrm{~Hz}, 1 \mathrm{H}), 7.76(\mathrm{dt}, J 1.5$ and $7.8 \mathrm{~Hz}, 1 \mathrm{H}), 8.12(\mathrm{dd}, J 1.5$ and $8.4 \mathrm{~Hz}, 1 \mathrm{H}), 8.22(\mathrm{dd}, J 1.5 \mathrm{and} 8.4 \mathrm{~Hz}$, $1 \mathrm{H}) ;{ }^{13} \mathrm{C}-\mathrm{NMR} \delta_{\mathrm{C}}\left(75 \mathrm{MHz}, \mathrm{CDCl}_{3}\right) 121.5,123.0,126.2,127.0,127.9,128.5,128.6,129.4,130.4$, 131.0, 131.5, 134.0, 134.2, 138.6, 142.6, 147.8, 158.2; $\mathrm{m} / z 329\left(100, \mathrm{MH}^{+}-\mathrm{N}_{2}\right)$, HRMS (ES): $\mathrm{MH}^{+}-\mathrm{N}_{2}$ found $329.0846 . \mathrm{C}_{21} \mathrm{H}_{14} \mathrm{~N}_{2}{ }^{35} \mathrm{Cl}^{+}$requires 329.0845 .

4-Amino-2-(4-chlorophenyl)-3-phenylquinoline (4c). Solid (130.7 mg, 64\%), mp 228-231 ${ }^{\circ} \mathrm{C}$ (ethanol) (Lit. [18] $\left.237-239^{\circ} \mathrm{C}\right), R_{\mathrm{F}} 0.09$. 
3.2.4. Biphenyl (2a), 4-Azido-2-(4-methoxyphenyl)-3-phenylquinoline (3d) and 4-Amino-2-(4-methoxyphenyl)-3-phenylquinoline (4d)

A mixture of $1 \mathbf{d}(0.30 \mathrm{~g}, 0.76 \mathrm{mmol})$, phenylboronic acid $(0.18 \mathrm{~g}, 1.49 \mathrm{mmol}), \mathrm{PdCl}_{2}\left(\mathrm{PPh}_{3}\right)_{2}(0.03 \mathrm{~g}$, $0.04 \mathrm{mmol}), \mathrm{PCy}_{3}(0.02 \mathrm{~g}, 0.07 \mathrm{mmol}), 2 \mathrm{M} \mathrm{K}_{2} \mathrm{CO}_{3}(1.5 \mathrm{~mL})$ in DMF (6 mL) was treated as described above. Work-up and column chromatography on silica gel (20\% ethyl acetate-hexane) afforded $\mathbf{2 a}$ $(0.02 \mathrm{~g}, 17 \%), R_{\mathrm{F}} 0.87, \mathbf{3 d}$ and $\mathbf{4 d}$ in sequence.

4-Azido-2-(4-methoxyphenyl)-3-phenylquinoline (3d). Solid (27.8 $\mathrm{mg}, 16 \%), \mathrm{mp} 135-136{ }^{\circ} \mathrm{C}$ (ethanol), $R_{\mathrm{F}} 0.56 ; v_{\max }$ (neat) 670, 743, 1030, 1247, 1504, $2110 \mathrm{~cm}^{-1} ;{ }^{1} \mathrm{H}-\mathrm{NMR} \delta_{\mathrm{H}}\left(300 \mathrm{MHz}, \mathrm{CDCl}_{3}\right)$ $3.76(\mathrm{~s}, 3 \mathrm{H}), 6.73(\mathrm{~d}, J 8.7 \mathrm{~Hz}, 2 \mathrm{H}), 7.19-7.22(\mathrm{~m}, 2 \mathrm{H}), 7.27-7.35(\mathrm{~m}, 5 \mathrm{H}), 7.64(\mathrm{dt}, J 1.5 \mathrm{and} 8.1 \mathrm{~Hz}$, $1 \mathrm{H}), 7.78(\mathrm{dt}, J 1.5$ and $8.7 \mathrm{~Hz}, 1 \mathrm{H}), 8.19$ (dd, $J 0.9$ and $8.1 \mathrm{~Hz}, 1 \mathrm{H}), 8.30$ (dd, $J 1.2$ and $7.8 \mathrm{~Hz}, 1 \mathrm{H}$ ); ${ }^{13} \mathrm{C}-\mathrm{NMR} \delta_{\mathrm{C}}\left(75 \mathrm{MHz}, \mathrm{CDCl}_{3}\right)$ 55.2, 113.2, 124.6, 125.3, 127.4, 127.6, 128.1, 129.7, 130.2, 130.7, $131.3,132.7,132.8,137.4,141.8,147.7,158.7,159.4 ; \mathrm{m} / z 353\left(30, \mathrm{MH}^{+}\right), 325.1346\left(100, \mathrm{MH}^{+}-\mathrm{N}_{2}\right)$, HRMS (ES): $\mathrm{MH}^{+}$found 353.1414. $\mathrm{C}_{22} \mathrm{H}_{17} \mathrm{~N}_{4} \mathrm{O}^{+}$requires 353.1402 .

4-Amino-2-(4-methoxyphenyl)-3-phenylquinoline (4d). Solid (160 mg, 66\%), mp 166-169 ${ }^{\circ} \mathrm{C}$ (ethanol) (Lit. [18] $\left.165-167^{\circ} \mathrm{C}\right), R_{\mathrm{F}} 0.10$.

3.2.5. 4,4'-Difluoro-1,1'-biphenyl (2b) and 4-Amino-3-(4-fluorophenyl)-2-(phenyl)quinoline (4e)

A mixture of $1 \mathrm{a}(0.20 \mathrm{~g}, 0.54 \mathrm{mmol})$, 4-fluorophenylboronic acid (0.15 g, $1.08 \mathrm{mmol}), \mathrm{PdCl}_{2}\left(\mathrm{PPh}_{3}\right)_{2}$ (0.02 g, $0.03 \mathrm{mmol}), \mathrm{PCy}_{3}(0.02 \mathrm{~g}, 0.05 \mathrm{mmol}), 2 \mathrm{M} \mathrm{K}_{2} \mathrm{CO}_{3}(1.2 \mathrm{~mL})$ in DMF $(6 \mathrm{~mL})$ was treated as described above. Work-up and column chromatography on silica gel (20\% ethyl acetate-hexane) afforded $2 b$ and $4 \mathbf{e}$ in sequence.

1-Fluoro-4-(4-fluorophenyl)benzene (2b). Solid (19.8 mg, 20\%), mp 85-87 ${ }^{\circ} \mathrm{C}$ (ethanol) (Lit. [35] $\left.87-88^{\circ} \mathrm{C}\right), R_{\mathrm{F}} 0.94$.

4-Amino-3-(4-fluorophenyl)-2-(phenyl)quinoline (4e). Solid (110 mg, 65\%), mp 243-245 ${ }^{\circ} \mathrm{C}$ (ethanol), $R_{\mathrm{F}} 0.15 ; v_{\max }$ (neat) 692, 756, 1365, 1612, $3392 \mathrm{~cm}^{-1} ;{ }^{1} \mathrm{H}-\mathrm{NMR} \delta_{\mathrm{H}}\left(300 \mathrm{MHz}, \mathrm{CDCl}_{3}\right) 4.71$ (br s, 2H), $7.03(\mathrm{t}, J 8.4 \mathrm{~Hz}, 2 \mathrm{H}), 7.14-7.21(\mathrm{~m}, 5 \mathrm{H}), 7.27-7.31(\mathrm{~m}, 2 \mathrm{H}), 7.49(\mathrm{dt}, J 1.5$ and $8.7 \mathrm{~Hz}, 1 \mathrm{H}), 7.69$ (dt, $J 1.5$ and $8.7 \mathrm{~Hz}, 1 \mathrm{H}), 7.78(\mathrm{dd}, J 0.6$ and $8.7 \mathrm{~Hz}, 1 \mathrm{H}), 8.10(\mathrm{dd}, J 0.6$ and $8.7 \mathrm{~Hz}, 1 \mathrm{H}) ;{ }^{13} \mathrm{C}-\mathrm{NMR} \delta_{\mathrm{C}}$ $\left(75 \mathrm{MHz}, \mathrm{CDCl}_{3}\right) 114.9,116.1\left(\mathrm{~d},{ }^{2} J_{\mathrm{CF}} 21.3 \mathrm{~Hz}\right), 117.4,120.3,125.2,127.4,127.9,129.5,129.6$, 130.2, $132.2\left(\mathrm{~d},{ }^{4} J_{\mathrm{CF}} 3.7 \mathrm{~Hz}\right), 132.8\left(\mathrm{~d},{ }^{3} J_{\mathrm{CF}} 8.3 \mathrm{~Hz}\right), 141.1,147.3,147.6,158.9,162.0\left(\mathrm{~d},{ }^{1} J_{\mathrm{CF}} 245.6 \mathrm{~Hz}\right)$; $m / z 315\left(100, \mathrm{MH}^{+}\right)$, HRMS (ES): $\mathrm{MH}^{+}$found 315.1301. $\mathrm{C}_{21} \mathrm{H}_{16} \mathrm{FN}_{2}$ requires 315.1293 .

\subsubsection{1-Fluoro-4-(4-fluorophenyl)benzene (2b) and 4-Amino-2,3-bis(4-fluorophenyl)quinoline (4f)}

A mixture of $\mathbf{1 b}(0.50 \mathrm{~g}, 1.28 \mathrm{mmol})$, 4-fluorophenylboronic acid (0.36 g, $2.56 \mathrm{mmol})$, $\mathrm{PdCl}_{2}\left(\mathrm{PPh}_{3}\right)_{2}(0.04 \mathrm{~g}, 0.06 \mathrm{mmol}), \mathrm{PCy}_{3}(0.04 \mathrm{~g}, 0.13 \mathrm{mmol}), 2 \mathrm{M} \mathrm{K}_{2} \mathrm{CO}_{3}(2.6 \mathrm{~mL})$ in DMF $(6 \mathrm{~mL})$ was treated as described above. Work-up and column chromatography on silica gel (40\% ethyl acetate-hexane) afforded $\mathbf{2 b}(30 \mathrm{mg}, 12 \%) R_{\mathrm{F}} 0.90$ and $\mathbf{4 f}$ in sequence. 
4-Amino-2,3-bis(4-fluorophenyl)quinoline (4f). Solid (280 mg, 66\%), mp 247-249 ${ }^{\circ} \mathrm{C}$ (ethanol), $R_{\mathrm{F}}$ $0.23 ; v_{\max }$ (neat) 616, 797, 881, 1494, 1561, 1616, 3308, $3435 \mathrm{~cm}^{-1} ;{ }^{1} \mathrm{H}-\mathrm{NMR} \delta_{\mathrm{H}}\left(300 \mathrm{MHz}, \mathrm{CDCl}_{3}\right)$ 4.73 (br s, 2H), 6.88 (t, J 8.7 Hz, 2H), 7.05 (t, J 8.7 Hz, 2H), 7.16 (t, J 8.7 Hz, 2H), 7.27 (t, J 8.7 Hz, 2H), 7.49 (dt, $J 1.2$ and $8.4 \mathrm{~Hz}, 1 \mathrm{H}), 7.68(\mathrm{dt}, J 1.5$ and $8.4 \mathrm{~Hz}, 1 \mathrm{H}), 7.78(\mathrm{dd}, J 1.2$ and $8.4 \mathrm{~Hz}, 1 \mathrm{H})$, $8.07(\mathrm{~d}, J 8.1 \mathrm{~Hz}, 1 \mathrm{H}) ;{ }^{13} \mathrm{C}-\mathrm{NMR} \delta_{\mathrm{C}}\left(75 \mathrm{MHz}, \mathrm{CDCl}_{3}\right) 114.6\left(\mathrm{~d},{ }^{2} J_{\mathrm{CF}} 21.3 \mathrm{~Hz}\right), 114.7,116.3\left(\mathrm{~d},{ }^{2} J_{\mathrm{CF}}\right.$ $21.4 \mathrm{~Hz}), 117.3,120.3,125.2,129.7,130.2,131.4\left(\mathrm{~d},{ }^{3} J_{\mathrm{CF}} 8.0 \mathrm{~Hz}\right), 132.2\left(\mathrm{~d},{ }^{4} J_{\mathrm{CF}} 3.5 \mathrm{~Hz}\right), 132.8(\mathrm{~d}$, $\left.{ }^{3} J_{\mathrm{CF}} 8.0 \mathrm{~Hz}\right), 137.2\left(\mathrm{~d},{ }^{4} J_{\mathrm{CF}} 3.5 \mathrm{~Hz}\right), 147.4,147.6,157.8,162.1\left(\mathrm{~d},{ }^{1} J_{\mathrm{CF}} 246.2 \mathrm{~Hz}\right), 162.2\left(\mathrm{~d},{ }^{1} J_{\mathrm{CF}} 245.6\right.$ $\mathrm{Hz}) ; m / z\left(100, \mathrm{MH}^{+}\right)$, HRMS (ES): $\mathrm{MH}^{+}$found 333.1207. $\mathrm{C}_{21} \mathrm{H}_{15} \mathrm{~F}_{2} \mathrm{~N}_{2}{ }^{+}$requires 333.1203.

\subsubsection{1-Fluoro-4-(4-fluorophenyl)benzene (2b) and} 4-Amino-2-(4-chlorophenyl)-3-(4-fluorophenyl)quinoline (4g)

A mixture of $1 \mathrm{c}(0.25 \mathrm{~g}, 0.62 \mathrm{mmol})$, 4-fluorophenylboronic acid (0.17 g, $1.23 \mathrm{mmol}), \mathrm{PdCl}_{2}\left(\mathrm{PPh}_{3}\right)_{2}$ $(0.02 \mathrm{~g}, 0.03 \mathrm{mmol}), \mathrm{PCy}_{3}(0.03 \mathrm{~g}, 0.06 \mathrm{mmol}), 2 \mathrm{M} \mathrm{K}_{2} \mathrm{CO}_{3}(1.2 \mathrm{~mL})$ in DMF $(8 \mathrm{~mL})$ was treated as described above. Work-up and column chromatography on silica gel (20\% ethyl acetate-hexane) afforded $\mathbf{2 b}(19.5 \mathrm{mg}, 16 \%), R_{\mathrm{F}} 0.88$ and $\mathbf{4 g}$ in sequence.

4-Amino-2-(4-chlorophenyl)-3-(4-fluorophenyl)quinoline (4g). Solid (141 mg, 65\%), mp 246-248 ${ }^{\circ} \mathrm{C}$ (ethanol), $R_{\mathrm{F}} 0.10 ; v_{\max }$ (neat) $762,833,1224,1431,1490,1616,3191,3310,3428 \mathrm{~cm}^{-1} ;{ }^{1} \mathrm{H}-\mathrm{NMR} \delta_{\mathrm{H}}$ (300 MHz, $\left.\mathrm{CDCl}_{3}\right) 4.73$ (br s, 2H), 7.07 (t, J 8.7 Hz, 2H), 7.15-7.20 (m, 3H), 7.24-7.27 (m, 3H), 7.51 $(\mathrm{dt}, J 1.5$ and $8.4 \mathrm{~Hz}, 1 \mathrm{H}), 7.71(\mathrm{dt}, J 1.5$ and $8.1 \mathrm{~Hz}, 1 \mathrm{H}), 7.79(\mathrm{dd}, J 0.6$ and $8.4 \mathrm{~Hz}, 1 \mathrm{H}), 8.08$ (dd, $J 0.6$ and $8.7 \mathrm{~Hz}, 1 \mathrm{H}) ;{ }^{13} \mathrm{C}-\mathrm{NMR} \delta_{\mathrm{C}}\left(75 \mathrm{MHz}, \mathrm{CDCl}_{3}\right) 114.7,116.3\left(\mathrm{~d},{ }^{2} J_{\mathrm{CF}} 21.3 \mathrm{~Hz}\right), 117.4,120.3$, 125.4, 127.9, 129.7, 130.2, 131.0, $132.1\left(\mathrm{~d},{ }^{4} J_{\mathrm{CF}} 3.7 \mathrm{~Hz}\right), 132.8\left(\mathrm{~d},{ }^{3} J_{\mathrm{CF}} 8.0 \mathrm{~Hz}\right), 133.6,139.6,147.4$, 147.6, 157.6, 162.1 (d, $\left.{ }^{1} J_{\mathrm{CF}} 246.5 \mathrm{~Hz}\right) ; \mathrm{m} / z 349\left(100, \mathrm{MH}^{+}\right.$), $\mathrm{HRMS}$ (ES): $\mathrm{MH}^{+}$found 349.0912. $\mathrm{C}_{21} \mathrm{H}_{15} \mathrm{FN}_{2}{ }^{35} \mathrm{Cl}^{+}$requires 349.0908 .

3.2.8. 1-Fluoro-4-(4-fluorophenyl)benzene (2b) and 4-Amino-3-(4-fluorophenyl)-2-(4-methoxyphenyl)quinoline (4h)

A mixture of 1d $(0.20 \mathrm{~g}, 0.50 \mathrm{mmol}), 4$-fluorophenylboronic acid (0.14 g, $0.99 \mathrm{mmol})$, $\mathrm{PdCl}_{2}\left(\mathrm{PPh}_{3}\right)_{2}(0.02 \mathrm{~g}, 0.02 \mathrm{mmol}), \mathrm{PCy}_{3}(0.02 \mathrm{~g}, 0.05 \mathrm{mmol}), 2 \mathrm{M} \mathrm{K}_{2} \mathrm{CO}_{3}(1.0 \mathrm{~mL})$ in DMF $(6 \mathrm{~mL})$ was treated as described above. Work-up and column chromatography on silica gel (20\% ethyl acetatehexane) afforded $\mathbf{2 b}(19.6 \mathrm{mg}, 21 \%), R_{\mathrm{F}} 0.93$ and $\mathbf{4 h}$ in sequence.

4-Amino-3-(4-fluorophenyl)-2-(4-methoxyphenyl)quinoline (4h). Solid (111 mg, 64\%), mp 236-240 ${ }^{\circ} \mathrm{C}$ (ethanol), $R_{\mathrm{F}} 0.12 ; v_{\max }$ (neat) $609,764,834,1211,1245,1591,1608,3412,3477 \mathrm{~cm}^{-1} ;{ }^{1} \mathrm{H}-\mathrm{NMR} \delta_{\mathrm{H}}$ $\left(300 \mathrm{MHz}, \mathrm{CDCl}_{3}\right) 3.75$ (s, 3H), 4.73 (br s, 2H), 7.07 (t, J 8.7 Hz, 2H), 7.15-7.20 (m, 4H), 7.24-7.27 $(\mathrm{m}, 2 \mathrm{H}), 7.51(\mathrm{t}, J 8.4 \mathrm{~Hz}, 1 \mathrm{H}), 7.71(\mathrm{t}, J 8.4 \mathrm{~Hz}, 1 \mathrm{H}), 7.79(\mathrm{dd}, J 0.6$ and $8.7 \mathrm{~Hz}, 1 \mathrm{H}), 8.08(\mathrm{dd}, J 0.6$ and $8.7 \mathrm{~Hz}, 1 \mathrm{H}) ;{ }^{13} \mathrm{C}-\mathrm{NMR} \delta_{\mathrm{C}}\left(75 \mathrm{MHz}, \mathrm{CDCl}_{3}\right) 55.2,113.1,114.8,116.2\left(\mathrm{~d},{ }^{2} J_{\mathrm{CF}} 21.3 \mathrm{~Hz}\right), 117.3$, 120.3, 124.9, 129.5, 130.1, 131.0, $132.6\left(\mathrm{~d},{ }^{4} J_{\mathrm{CF}} 3.2 \mathrm{~Hz}\right), 132.8\left(\mathrm{~d},{ }^{3} J_{\mathrm{CF}} 8.0 \mathrm{~Hz}\right), 133.6,147.2,147.6$, 158.4, 159.0, $162.0\left(\mathrm{~d},{ }^{1} J_{\mathrm{CF}} 245.6 \mathrm{~Hz}\right) ; \mathrm{m} / z 345\left(100, \mathrm{MH}^{+}\right)$, HRMS (ES): $\mathrm{MH}^{+}$found 345.1411. $\mathrm{C}_{22} \mathrm{H}_{18} \mathrm{FN}_{2} \mathrm{O}^{+}$requires 345.1403 . 
3.2.9. 1-Methoxy-4-(4-methoxyphenyl)benzene (2c) and

4-Amino-3-(4-methoxyphenyl)-2-phenylquinoline (4i)

A mixture of 1a $(0.20 \mathrm{~g}, 0.54 \mathrm{mmol})$, 4-methoxyphenylboronic acid $(0.16 \mathrm{~g}, 1.08 \mathrm{mmol})$, $\mathrm{PdCl}_{2}\left(\mathrm{PPh}_{3}\right)_{2}(0.02 \mathrm{~g}, 0.03 \mathrm{mmol}), \mathrm{PCy}_{3}(0.02 \mathrm{~g}, 0.05 \mathrm{mmol}), 2 \mathrm{M} \mathrm{K}_{2} \mathrm{CO}_{3}(1.0 \mathrm{~mL})$ in DMF $(5 \mathrm{~mL})$ was treated as described above. Work-up and column chromatography on silica gel (20\% ethyl acetate-hexane) afforded $\mathbf{2} \mathbf{c}$ and $\mathbf{4 i}$ in sequence.

1-Methoxy-4-(4-methoxyphenyl)benzene (2c). Solid (30 mg, 16\%), mp 179-181 ${ }^{\circ} \mathrm{C}$ (ethanol) (Lit. [35] $\left.178-180{ }^{\circ} \mathrm{C}\right), R_{\mathrm{F}} 0.63$.

4-Amino-3-(4-methoxyphenyl)-2-phenylquinoline (4i). Solid (110 mg, 63\%), mp 202-204 ${ }^{\circ} \mathrm{C}$ (ethanol), $R_{\mathrm{F}} 0.08 ; v_{\max }$ (neat) $704,765,1240,1440,1510,1558,1567,1607,3322,3440 \mathrm{~cm}^{-1} ;{ }^{1} \mathrm{H}-\mathrm{NMR} \delta_{\mathrm{H}}(300$ $\left.\mathrm{MHz} \mathrm{CDCl}_{3}\right) 3.79$ (s, 3H), 4.73 (br s, 2H), 6.87 (d, J 8.7 Hz, 2H), 7.11 (d, J 8.7 Hz, 2H), 7.16-7.20 $(\mathrm{m}, 3 \mathrm{H}), 7.17-7.35(\mathrm{~m}, 2 \mathrm{H}), 7.48(\mathrm{dt}, J 0.9$ and $7.8 \mathrm{~Hz}, 1 \mathrm{H}), 6.68(\mathrm{t}, J 0.9$ and $8.4 \mathrm{~Hz}, 1 \mathrm{H}), 7.78(\mathrm{dd}$, $J 0.6$ and $8.7 \mathrm{~Hz}, 1 \mathrm{H}), 8.10(\mathrm{dd}, J 0.6$ and $8.4 \mathrm{~Hz}, 1 \mathrm{H}) ;{ }^{13} \mathrm{C}-\mathrm{NMR} \delta_{\mathrm{C}}\left(75 \mathrm{MHz}, \mathrm{CDCl}_{3}\right) 55.2,100.0$, 114.4, 115.7, 117.5, 120.3, 125.0, 127.3, 127.6, 128.4, 129.3, 129.7, 130.1, 132.2, 141.4, 147.5, 158.7, 158.9; $m / z$ 327. (100, $\mathrm{MH}^{+}$), HRMS (ES): $\mathrm{MH}^{+}$found 327.1507. $\mathrm{C}_{22} \mathrm{H}_{19} \mathrm{~N}_{2} \mathrm{O}^{+}$requires 327.1497.

3.2.10. 1-Methoxy-4-(4-methoxyphenyl)benzene (2c) and

4-Amino-2-(4-fluorophenyl)-3-(4-methoxyphenyl)-quinoline (4j)

A mixture of $\mathbf{1 b}(0.50 \mathrm{~g}, 1.28 \mathrm{mmol})$, 4-methoxyphenylboronic acid $(0.39 \mathrm{~g}, 2.56 \mathrm{mmol})$, $\mathrm{PdCl}_{2}\left(\mathrm{PPh}_{3}\right)_{2}(0.04 \mathrm{~g}, 0.06 \mathrm{mmol}), \mathrm{PCy}_{3}(0.04 \mathrm{~g}, 0.13 \mathrm{mmol}), 2 \mathrm{M} \mathrm{K}_{2} \mathrm{CO}_{3}(2.6 \mathrm{~mL})$ and DMF $(6 \mathrm{~mL})$ was treated as described above. Work-up and column chromatography on silica gel (40\% ethyl acetate-hexane) afforded $\mathbf{2 c}(50 \mathrm{mg}, 18 \%), R_{\mathrm{F}} 0.85$ and $\mathbf{4 j}$ in sequence.

4-Amino-2-(4-fluorophenyl)-3-(4-methoxyphenyl)quinoline (4j). Solid (250 mg, 57\%), mp 190-192 ${ }^{\circ} \mathrm{C}$ (ethanol), $R_{\mathrm{F}} 0.10 ; v_{\max }$ (neat) $611,763,799,1245,1510,1558,1607,3285,3427 \mathrm{~cm}^{-1} ;{ }^{1} \mathrm{H}-\mathrm{NMR} \delta_{\mathrm{H}}$ $\left(300 \mathrm{MHz} \mathrm{CDCl}_{3}\right) 3.81(\mathrm{~s}, 3 \mathrm{H}), 4.74(\mathrm{br} \mathrm{s}, 2 \mathrm{H}), 6.87(\mathrm{t}, J 8.7 \mathrm{~Hz}, 2 \mathrm{H}), 6.88(\mathrm{~d}, J 8.7 \mathrm{~Hz}, 2 \mathrm{H}), 7.09$ (d, $J 8.7 \mathrm{~Hz}, 2 \mathrm{H}), 7.31(\mathrm{t}, J 8.7 \mathrm{~Hz}, 2 \mathrm{H}), 7.47(\mathrm{t}, J 8.4 \mathrm{~Hz}, 1 \mathrm{H}), 7.67(\mathrm{dt}, J 1.2$ and $8.4 \mathrm{~Hz}, 1 \mathrm{H}), 7.76(\mathrm{~d}$, $J 8.4 \mathrm{~Hz}, 1 \mathrm{H}), 8.06(\mathrm{dd}, J 0.6$ and $8.4 \mathrm{~Hz}, 1 \mathrm{H}) ;{ }^{13} \mathrm{C}-\mathrm{NMR} \delta_{\mathrm{C}}\left(75 \mathrm{MHz}, \mathrm{CDCl}_{3}\right) 55.2,114.5\left(\mathrm{~d},{ }^{2} J_{\mathrm{CF}} 21.3\right.$ $\mathrm{Hz}), 114.6,115.5,117.4,120.3,125.0,128.2,129.4,130.1,131.5\left(\mathrm{~d},{ }^{3} J_{\mathrm{CF}} 8.0 \mathrm{~Hz}\right), 132.1,137.5\left(\mathrm{~d},{ }^{4} J_{\mathrm{CF}}\right.$ $3.5 \mathrm{~Hz}), 147.4,147.6,157.9,158.8,162.2$ (d, $\left.{ }^{1} J_{\mathrm{CF}} 245.3 \mathrm{~Hz}\right) ; \mathrm{m} / z 345$ (100, $\mathrm{MH}^{+}$), HRMS (ES): $\mathrm{MH}^{+}$ found 345.1403. $\mathrm{C}_{22} \mathrm{H}_{18} \mathrm{FN}_{2} \mathrm{O}^{+}$requires 345.1411.

3.2.11. 1-Methoxy-4-(4-methoxyphenyl)benzene (2c) and 4-Amino-2-(4-chlorophenyl)-3-(4-methoxyphenyl)-quinoline (4k)

A mixture of $\mathbf{1 b}(0.30 \mathrm{~g}, 0.74 \mathrm{mmol})$, 4-methoxyphenylboronic acid $(0.22 \mathrm{~g}, 1.48 \mathrm{mmol})$, $\mathrm{PdCl}_{2}\left(\mathrm{PPh}_{3}\right)_{2}(0.03 \mathrm{~g}, 0.04 \mathrm{mmol}), \mathrm{PCy}_{3}(0.02 \mathrm{~g}, 0.07 \mathrm{mmol}), 2 \mathrm{M} \mathrm{K}_{2} \mathrm{CO}_{3}(1.5 \mathrm{~mL})$ in DMF $(6 \mathrm{~mL})$ was treated as described above. Work-up and column chromatography on silica gel (40\% ethyl acetate-hexane) afforded $2 \mathrm{c}(29.7 \mathrm{mg}, 19 \%), R_{\mathrm{F}} 0.91$ and $\mathbf{4 k}$ in sequence. 
4-Amino-2-(4-chlorophenyl)-3-(4-methoxyphenyl)quinoline (4k). Solid (160 mg, 60\%), mp 223-225 ${ }^{\circ} \mathrm{C}$ (ethanol), $R_{\mathrm{F}} 0.26 ; v_{\max }$ (neat) 763, 826, 1246, 1511, 1559, 1609, 1633, 3291, $3432 \mathrm{~cm}^{-1} ;{ }^{1} \mathrm{H}-\mathrm{NMR} \delta_{\mathrm{H}}$ $\left(300 \mathrm{MHz}, \mathrm{CDCl}_{3}\right) 3.82(\mathrm{~s}, 3 \mathrm{H}), 4.74$ (br s, 2H), 6.89 (d, J 8.4 Hz, 2H), 7.09 (d, J 8.4 Hz, 2H), 7.16 (d, $J 8.4 \mathrm{~Hz}, 2 \mathrm{H}), 7.27(\mathrm{~d}, J 8.4 \mathrm{~Hz}, 2 \mathrm{H}), 7.48(\mathrm{t}, J 7.5 \mathrm{~Hz}, 1 \mathrm{H}), 7.67$ (t, $J 7.5 \mathrm{~Hz}, 1 \mathrm{H}), 7.77$ (d, $J 8.4 \mathrm{~Hz}$, $1 \mathrm{H}), 8.06(\mathrm{~d}, J 8.4 \mathrm{~Hz}, 1 \mathrm{H}) ;{ }^{13} \mathrm{C}-\mathrm{NMR} \delta_{\mathrm{C}}\left(75 \mathrm{MHz} \mathrm{CDCl}_{3}\right) 55.2,114.6,115.5,117.5,120.3,125.2$, $127.8,120.1,129.4,130.1,131.1,132.1,133.4,139.9,147.5,147.6,157.7,158.9 ; \mathrm{m} / z\left(100, \mathrm{MH}^{+}\right)$, HRMS (ES): $\mathrm{MH}^{+}$found 361.1111. $\mathrm{C}_{22} \mathrm{H}_{18} \mathrm{~N}_{2} \mathrm{O}^{35} \mathrm{Cl}^{+}$requires 361.1103.

3.2.12. 1-Methoxy-4-(4-methoxyphenyl)benzene (2c), 4-Azido-2,3-bis(4-methoxyphenyl)quinoline (31) and 4-Amino-2,3-bis(4-methoxyphenyl)quinoline (4l)

A mixture of 1d $(0.20 \mathrm{~g}, 0.50 \mathrm{mmol})$, 4-methoxyphenylboronic acid $(0.15 \mathrm{~g}, 0.99 \mathrm{mmol})$, $\mathrm{PdCl}_{2}\left(\mathrm{PPh}_{3}\right)_{2}(0.02 \mathrm{~g}, 0.02 \mathrm{mmol}), \mathrm{PCy}_{3}(0.02 \mathrm{~g}, 0.05 \mathrm{mmol}), 2 \mathrm{M} \mathrm{K}_{2} \mathrm{CO}_{3}(1 \mathrm{~mL})$ in DMF $(6 \mathrm{~mL})$ was treated as described above. Work-up and column chromatography on silica gel (40\% ethyl acetate-hexane) afforded $2 \mathrm{c}(30 \mathrm{mg}, 28 \%), R_{\mathrm{F}} 0.88$ and $\mathbf{4 1}$ in sequence.

4-Azido-2,3-bis(4-methoxyphenyl)quinoline (3l). Solid (16.2 mg, 9\%), mp 138-140 ${ }^{\circ} \mathrm{C}$ (ethanol), $R_{\mathrm{F}}$ $0.46 ; v_{\max }$ (neat) $715,829,1030,1175,1243,1479,1607,2110 \mathrm{~cm}^{-1} ;{ }^{1} \mathrm{H}-\mathrm{NMR} \delta_{\mathrm{H}}\left(300 \mathrm{MHz}, \mathrm{CDCl}_{3}\right)$ $3.83(\mathrm{~s}, 2 \times 3 \mathrm{H}), 6.88(\mathrm{~d}, J 8.7 \mathrm{~Hz}, 1 \mathrm{H}), 6.94(\mathrm{~d}, J 8.7 \mathrm{~Hz}, 2 \mathrm{H}), 7.16(\mathrm{~d}, J 8.7 \mathrm{~Hz}, 1 \mathrm{H}), 7.22(\mathrm{~d}, J 8.7 \mathrm{~Hz}$, 2H), $7.47(\mathrm{~d}, J 8.7 \mathrm{~Hz}, 2 \mathrm{H}), 7.57$ (dt, $J 1.2$ and $8.4 \mathrm{~Hz}, 1 \mathrm{H}), 7.75(\mathrm{dt}, J 1.2$ and $8.4 \mathrm{~Hz}, 1 \mathrm{H}), 8.10$ (dd, $J 0.9$ and $8.7 \mathrm{~Hz}, 1 \mathrm{H}), 8.21(\mathrm{dd}, J 0.9$ and $8.7 \mathrm{~Hz}, 1 \mathrm{H}) ;{ }^{13} \mathrm{C}-\mathrm{NMR} \delta_{\mathrm{C}}\left(75 \mathrm{MHz}, \mathrm{CDCl}_{3}\right) 55.2,55.3$, 113.9, 114.1, 123.0, 126.9, 127.7, 128.0, 129.4, 130.3, 131.0, 132.6, 133.4, 138.8, 143.1, 147.7, 158.5, 158.6, 159.7; m/z $355\left(100, \mathrm{MH}^{+}-\mathrm{N}_{2}\right)$, HRMS (ES): $\mathrm{MH}^{+}-\mathrm{N}_{2}$ found 355.1449. $\mathrm{C}_{23} \mathrm{H}_{19} \mathrm{~N}_{2} \mathrm{O}_{2}{ }^{+}$requires 355.1447 .

4-Amino-2,3-bis(4-methoxyphenyl)quinoline (4l). Solid (120 mg, 68\%), mp 202-204 ${ }^{\circ} \mathrm{C}$ (ethanol), $R_{\mathrm{F}}$ $0.25 ; v_{\max }$ (neat) 758, 1108, 1177, 1241, 1605, 3399, $3488 \mathrm{~cm}^{-1} ;{ }^{1} \mathrm{H}-\mathrm{NMR} \delta_{\mathrm{H}}\left(300 \mathrm{MHz}, \mathrm{CDCl}_{3}\right) 3.75$ (s, 3H), 3.81 (s, 3H), 4.70 (br s, 2H), $6.71(\mathrm{~d}, J 8.7 \mathrm{~Hz}, 2 \mathrm{H}), 6.89$ (d, J 8.7 Hz, 2H), 7.11 (d, J 8.7 Hz, 2H), $7.28(\mathrm{~d}, J 9.0 \mathrm{~Hz}, 2 \mathrm{H}), 7.45(\mathrm{dt}, J 1.5$ and $8.4 \mathrm{~Hz}, 1 \mathrm{H}), 7.65(\mathrm{dt}, J 1.5$ and $8.4 \mathrm{~Hz}, 1 \mathrm{H}), 7.75(\mathrm{~d}$, $J 8.7 \mathrm{~Hz}, 1 \mathrm{H}), 8.07(\mathrm{dd}, J 0.6$ and $8.4 \mathrm{~Hz}, 1 \mathrm{H}) ;{ }^{13} \mathrm{C}-\mathrm{NMR} \delta_{\mathrm{C}}\left(75 \mathrm{MHz}, \mathrm{CDCl}_{3}\right) 55.1,55.2,113.0,114.5$, 115.6, 117.4, 120.3, 124.8, 128.7, 129.2, 130.0, 130.1, 132.2, 133.9, 147.4, 147.5, 158.5 (2xC), 158.7; MS (EI) $m / z 357\left(100, \mathrm{MH}^{+}\right)$; HRMS (ES): $\mathrm{MH}^{+}$found 357.1611. $\mathrm{C}_{23} \mathrm{H}_{21} \mathrm{~N}_{2} \mathrm{O}_{2}{ }^{+}$requires 357.1603.

\subsection{Typical Procedure for the Pd(OAc) ${ }_{2}$-Catalyzed Cross-Coupling Reactions of $\mathbf{1} \mathbf{c}, \mathbf{d}$ with $\mathrm{PhB}(\mathrm{OH})_{2}$}

\subsubsection{Biphenyl (2a), 4-Azido-2-(4'-chlorophenyl)-3-phenylquinoline (3c) and}

4-Amino-2-(4'-chloro-phenyl)quinoline (4c)

A mixture of $1 \mathrm{c}(0.25 \mathrm{~g}, 0.62 \mathrm{mmol})$, phenylboronic acid $(0.07 \mathrm{~g}, 1.23 \mathrm{mmol}), \mathrm{Pd}(\mathrm{OAc})_{2}(0.01 \mathrm{~g}$, $0.03 \mathrm{mmol})$ and $2 \mathrm{M} \mathrm{K}_{2} \mathrm{CO}_{3}(1.2 \mathrm{~mL})$ in DMF $(6 \mathrm{~mL})$ in a two-necked flask equipped with a stirrer bar, rubber septum and a condenser was degassed with argon for $10 \mathrm{~min}$. A balloon filled with argon was connected to the top of the condenser and the mixture was heated at $80-90{ }^{\circ} \mathrm{C}$ for $6 \mathrm{~h}$. The mixture was cooled to room temperature and then poured into ice-cold water. The product was taken-up into chloroform and the organic solution was washed with brine, dried (anhydrous $\mathrm{MgSO}_{4}$ ) and then 
evaporated under reduced pressure. The residue was purified by column chromatography on silca gel (20\% ethyl acetate-hexane) to afford three products $\mathbf{2 a}(19 \mathrm{mg}, 21 \%), R_{\mathrm{F}} 0.94 ; \mathbf{3 c}(29.8 \mathrm{mg}, 14 \%)$, $R_{\mathrm{F}} 0.50$ and $4 \mathrm{c}(60 \mathrm{mg}, 30 \%), R_{\mathrm{F}} 0.14$ in sequence.

\subsubsection{Biphenyl (2a), 4-Azido-2-(4'-methoxyphenyl)-3-phenylquinoline (3d) and} 4-Amino-2-(4'-methoxyphenyl)quinoline (4d)

A mixture of $1 \mathrm{~d}(0.20 \mathrm{~g}, 0.50 \mathrm{mmol})$, phenylboronic acid $(0.12 \mathrm{~g}, 0.10 \mathrm{mmol}), \mathrm{Pd}(\mathrm{OAc})_{2}(0.01 \mathrm{~g}$, $0.02 \mathrm{mmol})$ and $2 \mathrm{M} \mathrm{K}_{2} \mathrm{CO}_{3}(1.2 \mathrm{~mL})$ in DMF $(6 \mathrm{~mL})$ was treated as described above. Workup and column chromatography on silica gel (20\% ethyl acetate-hexane, v/v) yielded three products 2 a $(20 \mathrm{mg}, 26 \%), R_{\mathrm{F}} 0.89 ; \mathbf{3 d}(30 \mathrm{mg}, 14 \%), R_{\mathrm{F}} 0.56$ and $\mathbf{4 d}(80 \mathrm{mg}, 50 \%), R_{\mathrm{F}} 0.11$ in sequence.

\section{Conclusions}

In summary, the direct one-pot palladium-mediated coupling of 2-aryl-4-azido-3-iodoquinolines $\mathbf{1}$ with arylboronic acids and subsequent reduction of the azido group by the in situ generated palladium hydride represents a convenient synthetic strategy for the construction of primary 4-amino-2,3-diarylquinolines. The isolation of the symmetrical biaryl derivatives 2 and the observed in situ reduction of the azido to amino group using either $\mathrm{Pd}(\mathrm{OAc})_{2}, \mathrm{PdCl}_{2}\left(\mathrm{PPh}_{3}\right)_{2}$ or $\mathrm{Pd}\left(\mathrm{PPh}_{3}\right)_{4}$ as the $\mathrm{Pd}(0)$ catalyst sources provide further support for the involvement of palladium hydride in the reductive elimination step of the catalytic cycle leading to self-coupling of arylboronic acids [32]. At least in our opinion, the results observed in this investigation rule out the possibility of an in situ reduction of the azido group via Staudinger reaction with $\mathrm{PPh}_{3}$ generated from the catalyst [20] or possible hydrogenation using DMF/water mixture as previously proposed in the literature [29,30]. The versatility of this methodology can be extended to develop a streamlined approach to 2,3-disubstituted primary 4-aminoquinoline libraries and their annulated quinoline derivatives. Moreover, the biaryl scaffold represents a privileged structure for pharmaceutically important compounds [36-38].

\section{Acknowledgements}

Financial support from the University of South Africa (UNISA) and the National Research Foundation (NRF) in South Africa is gratefully acknowledged. MMM thanks UNISA for the postgraduate assistantship through the Grow Your Own Timber Project (GYOT S0800000112).

\section{Conflict of Interest}

The authors declare no conflict of interest.

\section{References and Notes}

1. Wang, M.; Liu, Y.; Huang, Z. Novel and convenient synthesis of polyfunctionalized quinolines, quinolones and their annulation reactions. Tetrahedron Lett. 2001, 42, 2553-2555.

2. Hadjeri, M.; Mariotte, A.; Boumendjel, A. Alkylation of 2-phenyl-4-quinolones: Synthetic and structural studies. Chem. Pharm. Bull. 2001, 49, 1352-1355. 
3. Xia, Y.; Yang, Z.; Xia, P.; Hackl, T.; Hamel, E.; Mauger, A.; Wu, J.; Lee, K.J. Antitumor agents. 211. Fluorinated 2-phenyl-4-quinolone derivatives as antimitotic antitumor agents $J$. Med. Chem. 2001, 44, 3932-3936.

4. De, S.K.; Gibbs, R.A. A mild and efficient one-step synthesis of quinolines. Tetrahedron Lett. 2005, 46, 1647-1649.

5. Moyer, M.P.F.; Weber, H.; Gross, J.L. Structure-activity relationships of imidazo[4,5-f]quinoline partial structures and analogs. Discovery of pyrazolo[3,4-f]quinoline derivatives as potent immunostimulants. J. Med. Chem. 1992, 35, 4595-4601.

6. Palacios, F.; de Retana, A.M.O.; Oyarzabal, J. A simple synthesis of 3-phosphonyl-4aminoquinolines from $\beta$-enaminophosphonates. Tetrahedron 1999, 55, 5947-5964.

7. Maguire, M.P.; Sheets, K.R.; McVety, K.; Spada, A.P.; Zilberstein, A. A new series of PDGF receptor tyrosine kinase inhibitors: 3-substituted quinoline derivatives. J. Med. Chem. 1994, 37, 2129-2137.

8. Pinard, E.; Alanine, A.; Bourson, A.; Büttelmann, B.; Heitz, M.-P.; Mutel, V.; Gill, R.; Trube, G.; Wyler, R. 4-Aminoquinolines as a novel class of NR1/2B subtype selective NMDA receptor antagonists. Bioorg. Med. Chem. Lett. 2002, 12, 2615-2619.

9. Paliakov, E.; Strekowski, L. Boron tribromide mediated debenzylation of benzylamino and benzyloxy groups. Tetrahedron Lett. 2004, 45, 4093-4095.

10. Strekowski, L.; Janda, L.; Lee, H. Synthesis of bis(2-arylquinolin-4-yl)amines by lithium bis(trimethylsilyl)amide-mediated cyclization of ketimines derived from 2-(trifluoromethyl)anilines and aryl methyl ketones. J. Org. Chem. 1997, 62, 4193-4196.

11. Mekheimer, R.A.; Elgemeie, G.H.; Kappe, T. Synthesis of some novel azido- and tetrazoloquinoline-3-carbonitriles and their conversion into 2,4-diaminoquinoline-3-carbonitriles. J. Chem. Res. 2005, 82-85.

12. Mphahlele, M.J.; Gheevargheese, O.; Makhubela, N.F.H. Unprecedented outcome of basepromoted Neber rearrangement of $O$-mesyl oxime of 2-aryl-1,2,3,4-tetrahydro-1-methylsulfonyl-4quinolone- synthesis of 4-amino-2-arylquinolines. Phosphor. Sulfur Silicon 2000, 166, 303-314.

13. Aizikovich, A.; Kuznetsov, V.; Gorohovsky, S.; Levy, A.; Meir, S.; Byk, G.; Gellerman, G. A new application of diphenylphosphorylazide (DPPA) reagent: Convenient transformations of quinolin-4-one, pyridin-4-one and quinazolin-4-one derivatives into the 4-azido and 4-amino counterparts. Tetrahedron Lett. 2004, 45, 4241-4243.

14. Paliakov, E.; Strekowski, L. Boron tribromide mediated debenzylation of benzylamino and benzyloxy groups. Tetrahedron Lett. 2004, 45, 4093-4095.

15. Jones, G. Comprehensive Heterocyclic Chemistry, Katritzky, A.R., Rees, A.R., Eds.; Pergamon: Oxford, UK, 1984; Volume 2, p. 395.

16. Yum, E.K.; Yang, O.-K.; Kang, S.K.; Cheon, H.G.; Kim, S.S.; Choi, J.-K. Synthesis of


Chem. Soc. 2004, 25, 1091-1094.

17. Mphahlele, M.J.; Mphahlele, M.M. One-pot palladium-catalyzed C-I and C-H bond activation and subsequent Suzuki-Miyaura cross-coupling of 2-aryl-3-iodo-4-(phenylamino)quinolines with arylboronic acids. Tetrahedron 2011, 67, 4689-4695. 
18. Mphahlele, M.J.; Mtshemla, V. 2-Aryl-4-azido-3-(bromo/iodo)quinolines as substrates for the synthesis of primary 4-amino-2,3-disubstituted quinoline derivatives. J. Heterocycl. Chem. 2008, $45,1343-1350$.

19. Stockmann, V.; Fiksdahl, A. Preparation of new pyrido[3,4-b]thienopyrroles and pyrido[4,3e] thienopyridazines. Tetrahedron 2008, 64, 7626-7632.

20. Pudlo, M.; Csányi, D.; Moreau, F.; Hajós, G.; Riedl, Z.; Sapi, J. First Suzuki-Miyaura type cross-coupling of ortho-azidobromobenzene with arylboronic acids and its application to the synthesis of fused aromatic indole-heterocycles. Tetrahedron 2007, 63, 10320-10329.

21. Joshangani, M.; Faramarzi, E.; Rafiee, E.; Daryanavard, M.; Xiao, J.; Baillie, C. Highly efficient Suzuki coupling using moderately bulky tolylphosphine ligands. J. Mol. Catal. 2007, 273, 310-315.

22. Kürti, L.; Czakó, B. Strategic Applications of Named Reactions in Organic Synthesis; Elsevier Academic Press: London, UK, 2005; p. 448.

23. Slagt, V.F.; de Vries, A.H.M.; de Vries, J.G.; Kellog, R.M. Practical aspects of carbon-carbon cross-coupling reactions using heteroarenes. Org. Process Res. Dev. 2010, 14, 30-47.

24. Amatore, C.; Jutand, A. Mechanistic and kinetic studies of palladium catalytic systems. J. Organomet. Chem. 1999, 576, 254-278.

25. Ali, N.M.; Chattopadhyah, S.K.; McKillop, A.; Perret-Gentil, R.M.; Ozturk, T.; Rebelo, R.A. A short new route to the pyrido[2,3,4-kl]acridine subunit common to pyridoacridine alkaloids of marine origin. J. Chem. Soc. Chem. Commun. 1992, 1453-1454.

26. Stadlbauer, W.; Laschober, R.; Kappe, T. Potential non-steroidal estrogens and antiestrogens. IV. Organic azides in heterocyclic synthesis. Part 13. Synthesis of aza- and diazacoumestrols via azido derivatives. Monatsh. Chem. 1991, 122, 853-861.

27. Haman, B.C.; Hartwig, J.F. Tandem ring-closing metathesis transannular cyclization as a route to hydroxylated pyrrolizidines. Asymmetric synthesis of (+)-Australine. J. Am. Chem. Soc. 1998, 120, 7369-7370.

28. Itoh, T.; Mase, T. Direct synthesis of hetero-biaryl compounds containing an unprotected $\mathrm{NH}_{2}$ group via Suzuki-Miyaura reaction. Tetrahedron Lett. 2005, 46, 3573-3577.

29. Wang, H.-S.; Wang, Y.-C.; Pan, Y.-M.; Zhao, S.-L.; Chen, Z.-F. Simultaneous reduction of nitroto amino-group in the palladium-catalyzed Suzuki cross-coupling reaction. Tetrahedron Lett. 2008, 49, 2634-2637.

30. Yu, J.Y.; Shreiner, S.; Vaska, L. Homogeneous catalytic production of hydrogen and other molecules from water-DMF solutions. Inorg. Chim. Acta 1990, 170, 145-147.

31. Foucher, N.; Ambroise, Y.; Cintrat, J.-C.; Doris, E.; Pillon, F.; Rousseau, B. Highly chemoselective hydrogenolysis of iodoarenes. J. Org. Chem. 2002, 67, 932-934.

32. Moreno-Maňas, M.; Pérez, M.; Pleixats, R. Palladium-catalyzed Suzuki-type self-coupling of arylboronic acids. A mechanistic study. J. Org. Chem. 1996, 61, 2346-2351.

33. Song, Z.Z.; Wong, H.N.C. Regiospecific synthesis of furan-3,4-diyl oligomers via palladiumcatalyzed self-coupling of organoboroxines. J. Org. Chem. 1994, 59, 33-41.

34. Hills, I.D.; Fu, G.C. Elucidating reactivity differences in palladium-catalyzed coupling processes: The chemistry of palladium hydrides. J. Am. Chem. Soc. 2004, 126, 13178-13179. 
35. Zeng, M.; Du, Y.; Qi, C.; Zuo, S.; Li, X.; Shao, L.; Zhang, X.-M. An efficient and recyclable heterogeneous palladium catalyst utilizing naturally abundant pearl shell waste. Green Chem. 2011, 13, 350-356.

36. Hajduk, P.J.; Bures, M.; Praestgaard, J.; Fesik, S.W. Privileged molecules for protein binding identified from NMR-based screening. J. Med. Chem. 2000, 43, 3443-3447.

37. Faghih, R.; Dwight, W.; Pan, J.B.; Fox, G.B.; Krueger, K.M.; Esbenshade, T.A.; McVey, J.M.; Marsh, K.; Bennani, Y.L.; Hancock, A.A. Synthesis and SAR of aminoalkoxy-biaryl-4carboxamides: Novel and selective histamine H3 receptor antagonists. Bioorg. Med. Chem. Lett. 2003, 13, 1325-1328.

38. Look, G.C.; Vacin, C.; Dias, T.M.; Ho, S.; Tran, T.H.; Lee, L.L.; Wiesner, C.; Fang, F.; Marra, A.; Westmacott, D.; et al. The discovery of biaryl acids and amides exhibiting antibacterial activity against Gram-positive bacteria. Bioorg. Med. Chem. Lett. 2004, 14, 1423-1426.

Sample Availability: Samples of the compounds 1-4 are available from the authors.

(C) 2011 by the authors; licensee MDPI, Basel, Switzerland. This article is an open access article distributed under the terms and conditions of the Creative Commons Attribution license (http://creativecommons.org/licenses/by/3.0/). 